\title{
Ladrilhamentos por Poliominós na Norma $l_{p}{ }^{*}$
}

\author{
Antonio Campello \\ Depto de Matemática Aplicada, IMECC, UNICAMP \\ 13083-859, Campinas, SP \\ E-mail: campello@ime.unicamp.br
}

\section{João E. Strapasson}

Faculdade de Ciências Aplicadas, UNICAMP

13484-350, Limeira, SP

E-mail: joao.strapasson@fca.unicamp.br

\author{
Grasiele C. Jorge \\ Instituto de Ciência e Tecnologia, UNIFESP \\ 12231-280, São José dos Campos, SP \\ E-mail: grasiele.jorge@unifesp.br
}

\section{Sueli I. R. Costa}

Departamento de Matemática, UNICAMP

13083-859, Campinas, SP

E-mail: sueli@ime.unicamp.br

Resumo: $O$ objetivo deste trabalho é estudar ladrilhamentos de $\mathbb{R}^{n}$ por poliominós associados à norma $l_{p}$. No caso $p=1$, a Conjectura de Golomb-Welch, em aberto por cerca de 44 anos, propõe que não existe ladrilhamento por tais poliominós, exceto em casos muito especiais. Aqui estamos interessados em discutir a existência de ladrilhamentos no caso geral $1 \leq p \leq \infty$. Apresentamos resultados não-existenciais de ladrilhamentos para diferentes valores de p, bem como um estudo da forma e das propriedades combinatórias dos poliominós associados.

Palavras-chave: Reticulados, Poliominós, Códigos Perfeitos, Métrica de Lee

\section{Introdução}

Um poliominó em $\mathbb{R}^{n}$ é uma figura conexa formada pela união finita de cubos de lado unitário de tal maneira que pares de cubo são disjuntos ou compartilham uma face (de dimensão $k<n$ ). Um poliominó pode ser construído através da união de cubos centrados em pontos de $\mathbb{Z}^{n}$ contidos em uma bola na norma $l_{p}$, como mostrado na Fig. 1 .
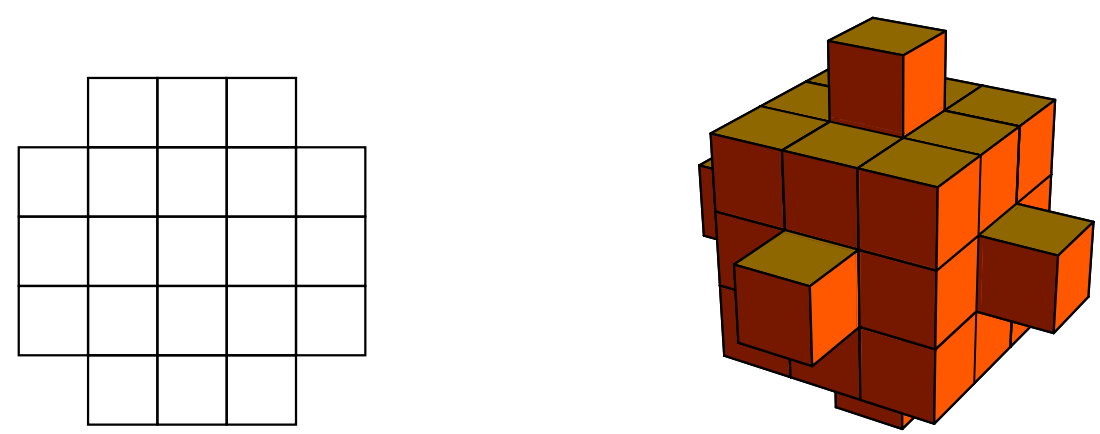

Figura 1: À esquerda, o poliominó $L_{2}(2, \sqrt{5})$ e à direita $L_{4}(3,2)$, conforme definido na Seção 2.1

Além do interesse puramente combinatório, o estudo de ladrilhamentos de $\mathbb{R}^{n}$ por poliominós surge naturalmente no contexto de códigos corretores de erros. A conexão mais antiga é através de códigos na métrica de Lee, proposta por Golomb e Welch em [4]. No artigo em questão, os autores mostram que códigos perfeitos na métrica de Lee em $\mathbb{Z}^{n}$ estão associados a ladrilhamentos de $\mathbb{R}^{n}$ por poliominós na norma $l_{1}$. A partir daí, é enunciada a seguinte conjectura: não existem códigos perfeitos na métrica de Lee para raio $R>1$ e $n>2$. Apesar de diversas tentativas e

\footnotetext{
*Este trabalho teve o apoio da FAPESP proc. 2011/22044-4 e 2011/01096-6 e do CNPQ proc. 150802/2012-9
} e 309561/2009-4 
resultados parciais (ver e.g., o recente artigo [5] e as referências nele contidas), o caso geral da Conjectura de Golomb-Welch encontra-se ainda em aberto. Mais recentemente, Schwartz [11] estuda a existência de ladrilhamentos por poliominós conhecidos como quasi-cruzes, os quais possuem aplicações no desenvolvimento de códigos para memórias flash.

Apesar de largamente estudada, e.g. $[4,5]$, a conjectura de Golomb-Welch só tem uma resposta definitiva para casos bem especiais (por exemplo, $n=2,3,4$ ). Assim, é de interesse considerar ladrilhamentos por estruturas que generalizam os poliominós considerados por Golomb e Welch em [4]. Algumas generalizações na literatura incluem o estudo de códigos quasiperfeitos, diametro-perfeito, e conjuntos perfeitamente dominantes. O objetivo deste artigo é estudar ladrilhamentos de $\mathbb{R}^{n}$ por poliominós associados a códigos perfeitos em $\mathbb{Z}^{n}$ na norma $l_{p}$.

O trabalho está organizado conforme descrito a seguir. Na Seção 2 descrevemos definições iniciais e resultados anteriores acerca do tema. Na Seção 3 analisamos a forma dos poliominós, descrevendo o raio crítico. Na Seção 3, estabelecemos um teorema assintótico de não-existência de ladrilhamentos, bem como resultados específicos para poliominós em $\mathbb{R}^{2}$ na norma $l_{2}$.

\section{Preliminares e Resultados Anteriores}

Seja $\mathbb{Z}^{n}$ o conjunto de todos os vetores cujas entradas são inteiras. Sejam $\boldsymbol{x}$ e $\boldsymbol{y}$ dois vetores de $\mathbb{Z}^{n}$ (ou $\mathbb{R}^{n}$ ). Denotamos a usual distância $l_{p}, 1 \leq p<\infty$ por

$$
d_{p}(\boldsymbol{x}, \boldsymbol{y})=\left(\sum_{i=1}^{n}\left|x_{i}-y_{i}\right|^{p}\right)^{1 / p} \text { e } d_{\infty}(\boldsymbol{x}, \boldsymbol{y})=\max \left\{\left|x_{i}-y_{i}\right| ; i=1, \ldots, n\right\}
$$

A norma $p\|\cdot\|_{p}$ correspondente é dada por $\|\boldsymbol{x}\|_{p}=d_{p}(\boldsymbol{x}, \mathbf{0})$. A bola fechada de raio $R$ centrada em $\boldsymbol{x}$ é dada por:

$$
B_{p}^{n}(\boldsymbol{x}, R)=\left\{\boldsymbol{y} \in \mathbb{R}^{n}: d_{p}(\boldsymbol{y}, \boldsymbol{x}) \leq R\right\} .
$$

Por simplicidade, a bola de raio $R$ centrada na origem será denotada por $B_{p}^{n}(R)$. Nosso objeto de estudo, um poliominó na norma $l_{p}$, é definido como a união de cubos de lado unitário centrados em pontos inteiros contidos em $B_{p}^{n}(R)$ (isto é, os pontos de $B_{p}^{n}(R) \cap \mathbb{Z}^{n}$ ). Mais formalmente, definimos o poliominó (associado à bola centrada na origem) $L_{p}(n, R)$ como:

$$
L_{p}(n, R)=\bigcup_{\boldsymbol{x} \in B_{p}^{n}(R) \cap \mathbb{Z}^{n}}\left(\boldsymbol{x}+\left[\frac{-1}{2}, \frac{1}{2}\right]^{n}\right) .
$$

Na Fig. 1 encontram-se ilustrados alguns poliominós em diferentes normas.

Uma boa parte do estudo de poliominós na literatura está interessada em saber quando um poliominó ladrilha ou não o $\mathbb{R}^{n}$. Seja $K \subset \mathbb{R}^{n}$ um conjunto de volume finito. Um conjunto de translações de $K$ com interiores disjuntos é dito um empacotamento de $K$. Se, adicionalmente, tal união é igual ao espaço inteiro $\mathbb{R}^{n}$, chamamos-na de ladrilhamento de $\mathbb{R}^{n}$. Se existe algum ladrilhamento de $\mathbb{R}^{n}$ por cópias de $K$, dizemos que $K$ ladrilha $\mathbb{R}^{n}$.

Conjuntos $K$ de interesse na literatura incluem poliominós [4, 11], politopos-cruz [3] (isto é $\left.K=\left\{\boldsymbol{x} \in \mathbb{R}^{n}:\|\boldsymbol{x}\|_{1}=R\right\}\right)$ e bolas na norma $l_{p}\left(K=B_{p}^{n}(R)\right)[10]$. Bolas na norma $l_{p}$ não ladrilham $\mathbb{R}^{n}$, exceto nos casos $p=1(n=2)$ e $p=\infty$ (qualquer $n$ ), portanto os problemas associados a esses conjuntos dizem respeito ao arranjo mais eficiente, ou com maior densidade de empacotamento, no espaço.

Alguns resultados sobre ladrilhamento por poliominós na norma $l_{p}$ encontram-se enunciados no que se segue.

Proposição 1. [4] O poliominó $L_{1}(2, R)$ ladrilha $\mathbb{R}^{2}$ para qualquer $R \geq 0$.

O próximo resultado foi provado em [4] para $p=1$ e em [8] para $1 \leq p<\infty$. 
Proposição $2([4,8])$. O poliominó $L_{p}(n, 1)$ ladrilha $\mathbb{R}^{n}$ para qualquer $n \geq 2$.

Conjectura (de Golomb-Welch [4]). Para $n>2$ e $R>1$, o poliominó $L_{1}(n, R)$ não ladrilha o $\mathbb{R}^{n}$.

Em [8], havia sido proposta uma extensão da conjectura de Golomb-Welch para as métricas $p$. De fato, essa conjectura é falsa se considerarmos valores de $R$ não-inteiros - permanecendo apenas o caso da métrica $l_{1}$. Mais precisamente, pode-se mostrar que para qualquer $n$ existe um $p_{0}$ tal que se $p>p_{0}$ o poliominó $L_{p}\left(n, R n^{1 / p}\right)$ ladrilha $\mathbb{R}^{n}$.

No que se segue revemos alguns conceitos sobre reticulados. Para maiores informações, sugerimos a referência [2]. Um reticulado (inteiro) $\Lambda \subset \mathbb{Z}^{n}$ é um conjunto da forma $\left\{\boldsymbol{u} B: \boldsymbol{u} \in \mathbb{Z}^{n}\right\}$, onde $B$ é uma matriz $n \times n$ não singular. Alternativamente, um reticulado é um subgrupo aditivo discreto de $\mathbb{Z}^{n}$. Dizemos também que um reticulado é um código (linear) em $\mathbb{Z}^{n}$. Apesar de a Teoria de Códigos estar usualmente relacionada a códigos no espaço vetorial $\mathbb{Z}_{q}^{n}, q$ primo, códigos em $\mathbb{Z}^{n}$ vem sendo estudados mais recentemente, principalmente no que tange a sua relação com códigos na métrica de Lee $[3,5]$.

A distância mínima $d_{p}(\Lambda)$ de um código $\Lambda \subset \mathbb{Z}^{n}$ na norma $l_{p}$ é definida como a menor distância entre dois elementos de $\Lambda$, ou equivalentemente, $d_{p}(\Lambda)=\min _{\boldsymbol{x} \in \Lambda \backslash\{\mathbf{0}\}}\|\boldsymbol{x}\|_{p}$. Um reticulado é um código perfeito (com raio $R$ ) na norma $l_{p}$ se existe $R>0$ tal que as bolas $B_{p}^{n}(\boldsymbol{x}, R) \cap \mathbb{Z}^{n}$ centradas em pontos $\boldsymbol{x} \in \Lambda$ são todas disjuntas duas a duas e cobrem todo o $\mathbb{Z}^{n}$. Alternativamente, um reticulado é um código perfeito se, e somente se, o poliominó $L_{p}(n, R)$ ladrilha o $\mathbb{R}^{n}$ por translações dos pontos de $\Lambda$.

Um reticulado induz um empacotamento em $\mathbb{R}^{n}$ por bolas na norma $l_{p}$ de raio $\rho=d(\Lambda) / 2$. A densidade de empacotamento de um reticulado é definida como $\Delta(\Lambda)=\operatorname{vol}\left(B_{p}^{n}(\rho)\right) /|\operatorname{det} B|$. Para $1<p<\infty$, a densidade é sempre um número estritamente menor que 1. Encontrar a maior densidade em $\mathbb{R}^{n}$ é um dos problemas centrais da teoria de reticulados [2].

\section{O Raio Crítico}

Para $p=1$ e $p=\infty$, é fácil ver que cada $R \in \mathbb{N}$ está associado a um poliominó distinto (isto é, $L_{1}\left(n, R_{1}\right) \neq L_{2}\left(n, R_{2}\right)$ se $R_{1}, R_{2} \in \mathbb{N}$ e $\left.R_{1} \neq R_{2}\right)$. Além disso, estes são os únicos raios em que ocorre uma mudança no tamanho do poliominó, isto é $L_{1}(n, R)=L_{1}(n, R+\varepsilon)$ para $0 \leq \varepsilon<1$. De uma certa forma, portanto, esses são os únicos raios relevantes para o estudo de tais poliominós. Para $1<p<\infty$ é possível que o poliominó mude de tamanho para raios que não são inteiros. Surge a necessidade de definir um raio crítico que, intuitivamente, é um raio tal que ocorre mudança de "tamanho" em $L_{p}(n, R)$. Dizemos, assim, que $R$ é um raio crítico de um poliominó na norma $l_{p}, 1 \leq p \leq \infty$ se $L_{p}(n, R-\varepsilon) \subsetneq L_{p}(n, R)$, para qualquer $\varepsilon>0$. Em outras palavras, um raio $R$ é crítico se existe $\boldsymbol{x} \in \mathbb{Z}^{n}$ tal que $\|\boldsymbol{x}\|_{p}=R$.

Denotemos por $\mathcal{R}(n, p)$ o conjunto de raios críticos de poliominós na norma $l_{p}$ em $\mathbb{Z}^{n}$. Segue facilmente da definição que $\mathcal{R}(n, p) \subset\left\{0,1^{1 / p}, 2^{1 / p}, 3^{1 / p}, \ldots\right\}$. Entretanto nem todo elemento tal que $R^{p}$ é inteiro é um raio crítico, como visto abaixo:

Exemplo 1. Tome $p=2$ e $n=2$. Temos que

$$
B_{2}^{2}(\sqrt{2}) \cap \mathbb{Z}^{2}=B_{2}^{2}(\sqrt{3}) \cap \mathbb{Z}^{2}=\{(0,0),( \pm 1,0),(0, \pm 1),( \pm 1, \pm 1)\} .
$$

Além disso $( \pm 1, \pm 1) \notin B_{2}(\sqrt{2}-\varepsilon) \cap \mathbb{Z}^{2}$ para qualquer $\varepsilon>0$. Portanto $\sqrt{2}$ é um raio crítico, enquanto $\sqrt{3}$ não é um raio crítico, apesar de ambos pertencerem ao conjunto $\left\{0,1^{1 / 2}, 2^{1 / 2}, 3^{1 / 2}, \ldots\right\}$.

Na norma $l_{2}$, podemos caraterizar completamente os raios críticos através de teoremas de soma de quadrados da Teoria dos Números.

Proposição 3. Um número $R \in\left\{0,1^{1 / p}, 2^{1 / p}, 3^{1 / p}, \ldots\right\}$ é um raio crítico com respeito a um poliominó na norma $l_{2}$ em $\mathbb{R}^{n}$ se, e somente se, 
(i) $n=2$ e $R^{2}$ pode ser escrito na forma $R^{2}=a b^{2}$, onde a não possui nenhum fator primo $q \equiv 3 \bmod 4 ;$ ou

(ii) $n=3$ e $R^{2}$ não é da forma $4^{m}(8 k+7)$, para $m, k \in \mathbb{N}$; ou

(iii) $n \geq 4$.

Demonstração. As propriedades acima são consequências diretas de teoremas da Teoria dos Números acerca de somas de quadrados. As afirmativas $(i)$ e (iii) são consequências do Teorema dos Dois Quadrados e Teorema dos Quatro Quadrados (veja, e.g., [7]). A propriedade (ii) segue do Teorema dos Três Quadrados, o qual pode ser encontrado (na forma em que necessitamos), em $[12$, p. 45].

Existe uma interessante conexão entre o raio crítico de um poliominó e o problema de Waring (e.g. [1]). Sejam $p \in \mathbb{N}, p \geq 2$. O problema de Waring pergunta pelo menor inteiro $k$ tal que qualquer número inteiro $m \in \mathbb{N}$ pode ser escrito como $m=x_{1}^{p}+\ldots+x_{k}^{p}$, pra $x_{i} \in \mathbb{N}$. O problema é bem definido [1] no sentido de que tal $k$ sempre existe.

Seja $g(p)$ a solução para o problema de Waring para $p \in \mathbb{N}$. Dos teoremas de somas de quadrados, temos claramente que $g(2)=4$. No survey [1] é apresentado o estado-da-arte no problema de Waring. Em particular, valem: $g(3)=9, g(4)=19$ e, sob algumas condições, $g(p)=2^{p}+\left\lfloor(3 / 2)^{p}\right\rceil-2$ (em especial para $k \leq 417.600 .000$ a fórmula é válida). A finitude de $g(p)$ implica diretamente a seguinte proposição:

Proposição 4. Para $n \geq g(p), \mathcal{R}(n, p)=\left\{0,1^{1 / p}, 2^{1 / p}, 3^{1 / p}, \ldots\right\}$.

Demonstração. Basta observar que se $R \in\left\{0,1^{1 / p}, 2^{1 / p}, 3^{1 / p}, \ldots\right\}$, então $R^{p}$ é inteiro, e assim pode ser escrito como a soma de $g(p)$ (ou mais) potências de $k$. Deste modo, $R$ é o raio de crítico de algum poliominó na norma $l_{p}$ em $\mathbb{Z}^{n}$.

\section{Teoremas de Não-Existência}

Seja $\mu_{p}(n, R)$ o número de pontos de $\mathbb{Z}^{n}$ na bola da distância $l_{p}$ com raio $R$, isto é

$$
\mu_{p}(n, R)=\#\left(\mathbb{Z}^{n} \bigcap B_{p}^{n}(R)\right),
$$

onde \# $M$ denota a cardinalidade de um conjunto finito $M$. Abaixo apresentamos uma demonstração que para $n$ e $p$ fixados e $R$ suficientemente grande o poliominó $L_{p}(n, R)$ não ladrilha $\mathbb{R}^{n}$. A ideia é estudar o empacotamento de bolas na norma $l_{p}$ associado a um código perfeito $\Lambda \subset \mathbb{Z}^{n}$. Para a nossa discussão, será necessário o seguinte lema, cuja demonstração pode ser obtida através dos mesmos argumentos que [9, pp. 134-135]

Lema 1. Seja $\Lambda$ um reticulado de posto completo com matriz geradora $B$ e seja $S(R)=\Lambda \cap$ $B_{p}^{n}(R)$ o conjunto dos pontos de $\Lambda$ contidos em $B_{p}^{n}(R)$. Temos:

$$
\sqrt{|\operatorname{det} B|}=\lim _{R \rightarrow \infty} \frac{\operatorname{vol}\left(B_{p}^{n}(R)\right)}{\# S(R)} .
$$

Teorema 1. Seja $n>2$ e $1 \leq p<\infty$. Existe $\bar{R}_{n, p}>0$ tal que para $R>\bar{R}_{n, p}$ não há códigos perfeitos lineares $\Lambda \subset \mathbb{Z}^{n}$ com raio $R$.

Demonstração. Suponhamos, por absurdo, que existe um código perfeito com raio $R$. O volume do poliominó $L_{p}(n, R)$ é igual a $\mu_{p}(n, R)$ e as cópias de $L_{p}(n, R)$ por pontos de $\Lambda$ ladrilham $\mathbb{R}^{n}$. 
Por outro lado, o raio de empacotamento do reticulado $\Lambda$ na norma $l_{p}$ é certamente maior ou igual a $R-\varepsilon$ (para algum $\varepsilon$ arbitrariamente pequeno) e portanto possui densidade:

$$
\delta \geq \frac{\operatorname{vol}\left(B_{p}^{n}(R-\varepsilon)\right)}{\operatorname{vol}\left(L_{p}(n, R)\right)}=\frac{\operatorname{vol}\left(B_{p}^{n}(R-\varepsilon)\right)}{\mu_{p}(n, R)} .
$$

Sabemos que o lado direito da desigualdade acima tende a $\operatorname{det} I_{n}=1$ quando $R$ tende a infinito (Lema 1). Mas também sabemos que a densidade de empacotamento de qualquer reticulado em $\mathbb{R}^{n}$ na norma $l_{p}$ é limitada superiormente por algum $\alpha<1$. Assim, para $R$ suficientemente grande, construímos um empacotamento que ultrapassa a melhor densidade de empacotamento em $\mathbb{R}^{n}$, o que é um absurdo, concluindo a demonstração.

Acima analisamos o poliominós $L_{p}(n, R)$ para $n$ e $p$ fixo, variando $R$. A partir de agora, faremos uma análise distinta, na qual permitimos variar o valor de $p$. Uma questão interessante é a existência ou não existência de ladrilhamentos para valores de $p>1$.

Definição 1. Seja $L_{p}(n, R)$ o poliominó dado por (2). Seja a um número inteiro e considere $Z_{a}=\mathbb{Z}^{n} \cap[-a, a]^{n}$ o conjunto de pontos inteiros contidos no cubo de lado 2 a centrado na origem.

- Se $B_{p}^{n}(R) \cap \mathbb{Z}^{n}=Z_{a}$, para algum a, dizemos que $L_{p}(n, R)$ possui formato $A$.

- Se $\left\{\boldsymbol{e}_{1}, \ldots, \boldsymbol{e}_{n}\right\}$ é a base canônica do $\mathbb{R}^{n}$ e

$$
B_{p}^{n}(R) \cap \mathbb{Z}^{n}=Z_{a} \bigcup( \pm(a+1)) \boldsymbol{e}_{1} \bigcup( \pm(a+1)) \boldsymbol{e}_{2} \ldots \bigcup( \pm(a+1)) \boldsymbol{e}_{n}
$$

para algum a, dizemos que $L_{p}(n, R)$ possui formato $B$.

- Se $B_{p}^{n}(R) \cap \mathbb{Z}^{n}=Z_{a} \backslash\left\{\left(x_{1}, \ldots, x_{n}\right): x_{i}= \pm a, i=1, \ldots n\right\}$ para algum a, dizemos que $L_{p}(n, R)$ possui formato $C$.

Segue imediatamente da definição que $L_{\infty}(n, R)$ possui formato A, com $a=\lfloor R\rfloor$. Além disso, o número de pontos em $B_{p}^{n}(R) \cap \mathbb{Z}^{n}$ para poliominós do tipo $A, B$ e $C$ é, respectivamente, $(2 a+1)^{n},(2 a+1)^{n}+2 n$ e $(2 a+1)^{n}-2^{n}$. Na proposição seguinte identificamos os três padrões de poliominós definidos acima.

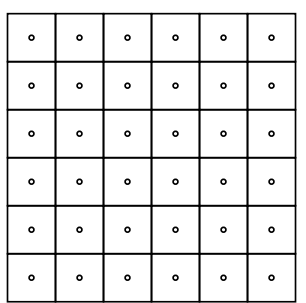

Formato A

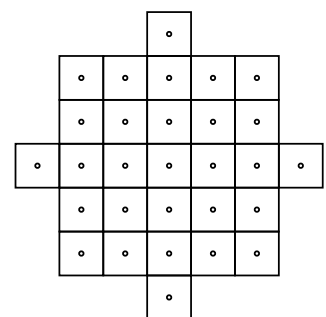

Formato B

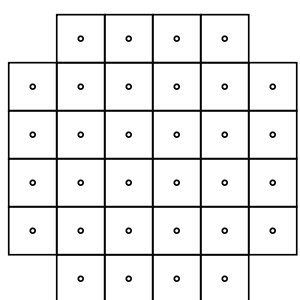

Formato $\mathrm{C}$

Proposição 5. Fixados um raio crítico $R \subset \mathcal{R}(n, p)$ e uma dimensão $n$, temos que:

(i) Se $R$ é inteiro e $\frac{\ln n}{\ln \left(\frac{R}{R-1}\right)} \leq p<\infty$, então $L_{p}(n, R)$ tem formato $B$, com $a=R-1$.

(ii) Se $R$ não for inteiro $e \frac{\ln n}{\ln \left(\frac{R}{[R]}\right)} \leq p<\infty$, então $L_{p}(n, R)$ tem o formato $A$, com $a=\lfloor R\rfloor$.

(iii) Se p é tal que $\left((n-1)\lfloor R\rfloor^{p}+(\lfloor R\rfloor-1)^{p}\right)^{1 / p} \leq R \leq n^{1 / p}\lfloor R\rfloor$, então $L_{p}(n, R)$ tem o formato $C, \operatorname{com} \operatorname{com} a=\lfloor R\rfloor$.

Demonstração. (i) Se $R$ é inteiro, note que $B_{\infty}^{n}(R-1) \subseteq B_{p}^{n}(R) \subseteq B_{\infty}^{n}(R)$ se, e somente se, $p \geq \frac{\ln n}{\ln \left(\frac{R}{R-1}\right)}$. De fato, a segunda inclusão sempre acontece independente do valor de $p$. Para a primeira inclusão temos: se $x \in B_{\infty}^{n}(R-1)$, então $\|x\|_{p} \leq n^{1 / p}\|x\|_{\infty} \leq n^{1 / p}(R-1) \leq R$ se, e somente se, $n^{1 / p} \leq \frac{R}{R-1}$, de onde segue o resultado. 
(ii) Se $R$ não é inteiro, então $B_{\infty}^{n}(\lfloor R\rfloor) \subseteq B_{p}^{n}(R) \subseteq B_{\infty}^{n}(\lceil R\rceil)$ se, e somente se, $\frac{\ln n}{\ln \left(\frac{R}{[R]}\right)} \leq p<$ $\infty$. Daí, para todo $p \geq \frac{\ln n}{\ln \left(\frac{R}{[R]}\right)}$, o poliominó tem formato A.

(iii) Para que a bola na métrica $p$ deixe apenas os cantos de fora, seu raio deve ser um pouco maior que a distância da origem até o ponto $(\lfloor R\rfloor,\lfloor R\rfloor, \cdots,\lfloor R\rfloor,\lfloor R\rfloor-1)$ que é $\left((n-1)\lfloor R\rfloor^{p}+(\lfloor R\rfloor-1)^{p}\right)^{1 / p}$ e um pouco menor que a distância da origem até o canto $(\lfloor R\rfloor,\lfloor R\rfloor, \cdots,\lfloor R\rfloor,\lfloor R\rfloor)$ que é $n^{1 / p}\lfloor R\rfloor$.

\subsection{Estimativas para $\bar{R}_{2,2}$}

Utilizando a demonstração do Teorema 1, podemos estimar quais são os raios a partir dos quais não há códigos perfeitos. No caso $\bar{R}_{n, 2}$ temos que conhecer essencialmente duas grandezas: a densidade do melhor empacotamento em dimensão $n, \Delta_{n}$, e uma fórmula (ao menos aproximada) para $\mu_{2}(n, R)$ (do Lema 1 , segue que $\mu_{2}(n, R) \approx \pi R^{2}$ para $R$ grande). O problema de determinar $\mu_{2}(n, R)$ é conhecido como o Problema do Círculo de Gauss. Argumentos elementares podem ser usados para mostrar que:

$$
\mu_{2}(n, R)=\pi R^{2}+E(R), \text { onde }|E(R)| \leq 2 \sqrt{2} \pi R
$$

Resultados mais recentes melhoram essa estimativa para $E(R) \leq C R^{\theta}$, para alguma constante $C$. O melhor expoente $\theta$ conseguido até então é aproximadamente 0.62981 devido a Huxley [6], entretanto na prática a constante $C$ pode ser muito grande. Utilizando a estimativa (4), e os argumentos da demonstração do teorema acima, vemos que a equação (3) ultrapassa $\Delta_{2}=$ $\pi / 3 \sqrt{2}$ ) (densidade o empacotamento hexagonal [2, p.15]) para $R \geq 45.1973$. Assim, como consequência do Teorema 1 para $R \geq 46$ o poliominó $L_{2}(2, R)$ não ladrilha $\mathbb{R}^{2}$.

Apesar de o limitante (4) poder ser usado para demonstrar que que $\bar{R}_{2,2} \leq 46$, experimentos computacionais sugerem que esta fórmula pode ser aprimorada. No gráfico abaixo, vemos que para $R>19$, a equação (3) viola a maior densidade de empacotamento possível.

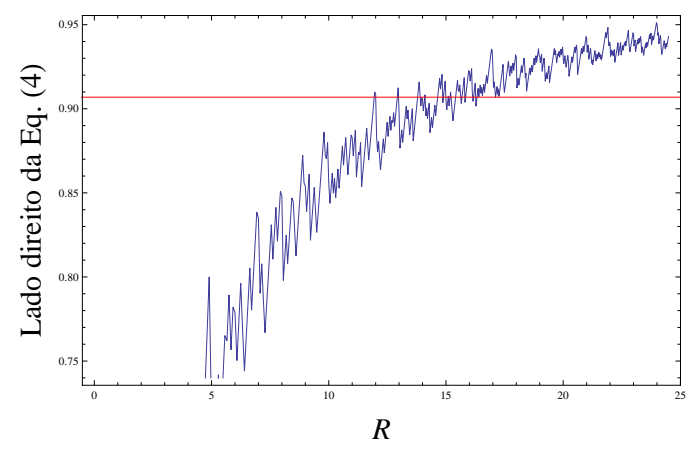

Figura 2: Experimentos para determinação de $\bar{R}_{2,2}$. A linha vermelha refere-se ao valor $\Delta_{2}=$ $\pi / 3 \sqrt{2}$ ), melhor densidade de empacotamento no plano.

\section{Conclusões e Perspectivas Futuras}

Os poliominós definidos pelo Caso (i) da Proposição 5 sugerem que não existem códigos perfeitos com raio inteiro para valores de $p$ suficientemente grandes. Para o raio crítico $R=2$ e $n=2$ existem códigos perfeitos com tal formato para $1 \leq p<\infty$, como pode ser visto na Figura 3 . Entretanto, à medida que aumentamos o raio, o Teorema 1 afirma que não há códigos perfeitos para valores de raio crítico suficientemente grande. A determinação precisa dos menores valores de $R$ e $p$ que não admitem códigos perfeitos, bem como demonstrações analíticas que poliominós com determinados formatos específicos não ladrilham $\mathbb{R}^{n}$ são perspectivas de trabalhos futuros. 


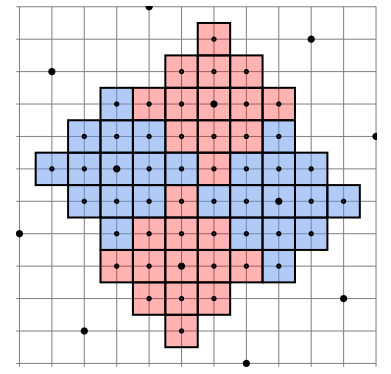

Figura 3: $L_{p}(2,2), 1 \leq p<\infty$

\section{Referências}

[1] Waring's problem: a survey. Number Theory for the Millennium, III (Urbana, IL, 2000), pages 301-340, 2012.

[2] J. H. Conway and N. J. A. Sloane. Sphere-packings, lattices, and groups. Springer-Verlag, New York, NY, USA, 1998.

[3] T. Etzion, A. Vardy, and E. Yaakobi. Dense error-correcting codes in the Lee metric. In IEEE Information Theory Workshop (ITW), pages 1-5, Aug 2010.

[4] S.W. Golomb and L. R. Welch. Perfect Codes in the Lee Metric and the Packing of Polyominoes. SIAM Journal on Applied Mathematics, 18(2):302-317, 1970.

[5] Peter Horak and Otokar Grosek. A new approach towards the Golomb-Welch conjecture. European Journal of Combinatorics, 38(0):12 - 22, 2014.

[6] M. N. Huxley. Exponential sums and lattice points iii. Proceedings of the London Mathematical Society, 87:591-609, 112003.

[7] H. L. Montgomery I. Niven, H. S. Zuckerman. An Introduction to The Theory of Numbers. Wiley.

[8] G. C. Jorge, A. Campello, and S. I. R. Costa. $q$-ary lattices in the $l_{p}$ norm and a generalization of the Lee metric. In Workshop on Coding and Cryptography (WCC), Bergen, Norway, 2013.

[9] (Editors) J.P. Buhler, P. Stevenhagen. Algorithmic Number Theory: Lattices. Number Fields, Curves and Cryptography, vol. 44, Cambridge University Press.

[10] J. A. Rush and N. J. A. Sloane. An improvement to the Minkowski-Hlawka bound for packing superballs. Mathematikal, 34:8-18, 1987.

[11] Moshe Schwartz. On the non-existence of lattice tilings by quasi-crosses. European Journal of Combinatorics, 36(0):130 - 142, 2014.

[12] J-P. Serre. A Course in Arithmetic. Graduate Texts in Mathematics, Vol. 7, Springer, 3 edition, 1996. 